\title{
On-Chip Arrayed Waveguide Grating Interrogated Silicon-on-Insulator Microring Resonator-Based Gas Sensor
}

\author{
Nebiyu Adello Yebo, Student Member, IEEE, Wim Bogaerts, Member, IEEE, Zeger Hens, and
} Roel Baets, Fellow, IEEE

\begin{abstract}
We demonstrate on-chip interrogation of a silicon-on-insulator microring resonator-based gas sensor with a compact arrayed waveguide grating (AWG) spectrometer. A 200-GHz silicon-on-insulator AWG with strongly overlapping output channels is used to interrogate a microring resonator sensor response for 100-1000 ppm ethanol vapor concentrations. Such an integrated sensor-interrogator approach is presented as an alternative to the current costly and off-chip read-out systems used for ring resonator-based sensors.
\end{abstract}

Index Terms-Arrayed waveguide grating (AWG), gas sensor, integrated, on-chip interrogation, photonic, ring resonator, silicon-on-insulator (SOI), zinc oxide.

\section{INTRODUCTION}

$\mathbf{P}$ HOTONIC integrated chemical and gas sensors have recently drawn a considerable interest due to their merits such as high compactness, low energy consumption, and low cost. Exploiting the advantages of the well-established semiconductor fabrication technology, Silicon-on-Insulator (SOI) micro-optical structures and devices, in particular, are proving to be highly suitable for a wide range of applications including sensing. Microring resonators (MRRs) are a type of SOI structures which have gained a significant attention for sensing owing to features such as high sensitivity to the surrounding refractive index variations, readiness for wavelength multiplexing, and compactness [1]-[3]. Yet, there are some issues which need proper technological solutions so as to ensure the feasibility of MRRs for sensing applications. One of the current limitations is the cost of the light sources or spectrum analyzers used to drive and interrogate MRR-based sensors. Either expensive tunable laser sources or spectrum analyzers are often used today [1]-[3]. One approach to address such an issue is to look for on-chip spectrometers which will work along with cheap broadband light sources instead of tunable lasers. Integrated Planar Concave Gratings (PCGs) and Array Waveguide Gratings (AWGs) have been widely studied

Manuscript received March 29, 2011; revised May 16, 2011; accepted July 15, 2011. Date of publication July 25, 2011; date of current version September 23,2011 . This work was funded by Ghent University under the framework of the "Methusalem Smart photonic chips" project.

N. A. Yebo, W. Bogaerts, and R. Baets are with the Photonics Research Group, Department of Information Technology, Ghent University-IMEC, 9000 Gent, Belgium (e-mail: nyebo@intec.ugent.be).

Z. Hens is with the Physics and Chemistry of Nanostructures Group, Ghent University, 9000 Gent, Belgium.

Color versions of one or more of the figures in this letter are available online at http://ieeexplore.ieee.org.

Digital Object Identifier 10.1109/LPT.2011.2162825 and used for wavelength division multiplexing applications [4]. In contrast to PCGs, narrow channel spacing AWGs can be realized on much smaller footprints on an SOI platform [4]. Different techniques based on AWGs have been recently reported for wavelength shift interrogation in Fiber Bragg Grating sensors [5]-[7]. To our knowledge, in previous demonstrations, either silica-based AWGs or larger footprint high resolution SOI AWGs were used. Moreover, the application of such interrogators for on-chip sensors such as ring resonators has not been yet demonstrated. By monitoring the intensity ratios between two adjacent channels of a high resolution, $0.18 \mathrm{~nm}$ channel spacing SOI AWG, it has been demonstrated that very small wavelength shifts from an FBG sensor can be interrogated [6]. However, such a high resolution comes at the cost of a large device size $(8 \mathrm{~mm} \times 8 \mathrm{~mm})$, and the reported insertion loss is very high. In addition, for very sensitive sensors, where large resonance shifts can be achieved at low analyte concentrations, moderate channel resolutions offer a better dynamic range for interrogation with intensity ratios. In this letter, we demonstrate that a $1.6 \mathrm{~nm}$ channel spacing AWG with broad spectral response can be used to interrogate very sensitive MRR-based gas sensors. The AWG used in this work takes a footprint of $500 \mu \mathrm{m} \times 200 \mu \mathrm{m}$ and is fabricated on a chip which also contains the MRR sensor.

In this work, we use an AWG to interrogate our recently reported MRR-based ethanol vapor sensor [3]. The ring is coated with a porous $\mathrm{ZnO}$ nanoparticle film. Ethanol vapor concentrations ranging from $100 \mathrm{ppm}$ to $1000 \mathrm{ppm}$ are readily monitored with our sensor-interrogator system by measuring the intensity ratios between adjacent AWG channels.

\section{SENSOR INTERROGATION BASICS}

In an AWG-interrogated MRR sensor configuration, the drop port of the microring resonator is connected to an AWG input port. The AWG is designed such that its adjacent output channels overlap significantly, so the MRR resonance will consequently be detected at any two of such overlapping channels. Ultimately, the variations in the intensity ratio between the adjacent channels can indicate the MRR resonance shift due to environmental effects.

Approximating the MRR resonance and the AWG channel response by Lorentzian and Gaussian profiles, respectively, (1) can be used to estimate the variations in intensity ratios between the $\mathrm{k}^{\mathrm{th}}$ and $(\mathrm{k}+1)^{\mathrm{th}}$ channels with the resonance shift [5], [6]. For instance, provided a $0.05 \mathrm{~nm}$ full width at half maximum (FWHM) resonance peak and a $200 \mathrm{GHz}$ AWG, Fig. 1 shows 


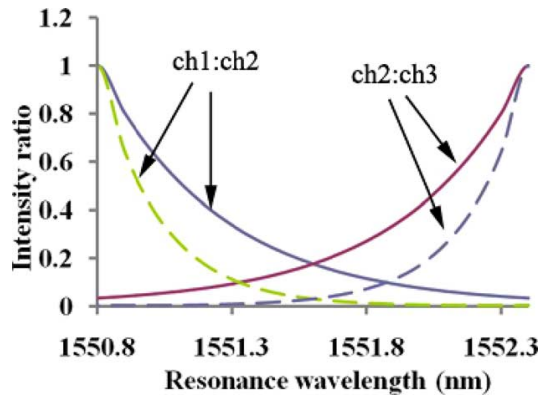

Fig. 1. Given three neighboring AWG channels centered at 1550, 1551.6, and $1553.2 \mathrm{~nm}$, theoretical estimates of intensity ratios between adjacent channels as a function of the MRR resonance wavelength for Gaussian profiles of $1.5 \mathrm{~nm}$ FWHM (dotted lines) and $2 \mathrm{~nm}$ FWHM (solid lines).

how the intensity ratio evolves across three neighboring AWG channels centered at $1550 \mathrm{~nm}, 1551.6 \mathrm{~nm}$ and $1553.2 \mathrm{~nm}$, as a function of the resonance wavelength. The solid and dotted curves show values calculated for Gaussian channel profiles with FWHM of $2 \mathrm{~nm}$ and $1.5 \mathrm{~nm}$, respectively. Most importantly, Fig. 1 demonstrates that a sufficient channel overlap is crucial in order to achieve well-resolvable power ratio changes corresponding to small resonance shifts. This criterion is more critical for a MRR with a very narrow resonance peak, and the effect becomes apparent as the resonance shifts further towards the center of a channel.

$$
\frac{I_{k}}{I_{k+1}}=\frac{\int_{0}^{\infty} T_{M R R}(\lambda) \cdot T_{A W G}(k, \lambda) d \lambda}{\int_{0}^{\infty} T_{M R R}(\lambda) \cdot T_{A W G}(k+1, \lambda) d \lambda} .
$$

Where, $T_{M R R}(\lambda), T_{A W G}(k, \lambda), T_{A W G}(k+1, \lambda)$ represent the transmissions from the MRR drop port, $\mathrm{k}^{\text {th }}$ AWG channel and $(\mathrm{k}+1)^{\mathrm{th}}$ AWG channel respectively, at an arbitrary wavelength, $\lambda$.

\section{DESIGN AND FABRICATION}

Different techniques such as multimode interference (MMI)coupled AWGs have been reported to provide a broad as well as flat channel response. For instance, the combined effect of broad and two-fold image of the input light produced by an MMI input waveguide has been utilized to obtain the above mentioned response [8]. For wavelength shift interrogation, fairly sharp response instead of a flat one is required while maintaining an adequate channel overlap. In such an application, the MMI can be replaced by a simple broad waveguide to obtain broader channel profiles. In this work, a 16 channel, $200 \mathrm{GHz}(1.6 \mathrm{~nm}$ channel spacing), AWG is designed such that the output from a $5 \mu \mathrm{m}$ radius MRR drop port overlaps with any two adjacent channels. Very compact and low insertion loss SOI AWGs were demonstrated in the past [4]. For this particular sensor interrogation application, an adiabatically tapered input waveguide with start width of $0.45 \mu \mathrm{m}$ and a broader end width of $4 \mu \mathrm{m}$ is chosen in order to broaden the channel response, and hence, enhance the overlap between adjacent channels. The start width of the tapered output waveguides is $2 \mu \mathrm{m}$.

The device is fabricated using $193 \mathrm{~nm}$ deep ultra violet (UV) lithography as detailed in [4]. To add gas sensitive functionality to the device, about $240 \mathrm{~nm}$ thick porous $\mathrm{ZnO}$ film is locally coated on the MRR. The $\mathrm{ZnO}$ film is prepared from col-

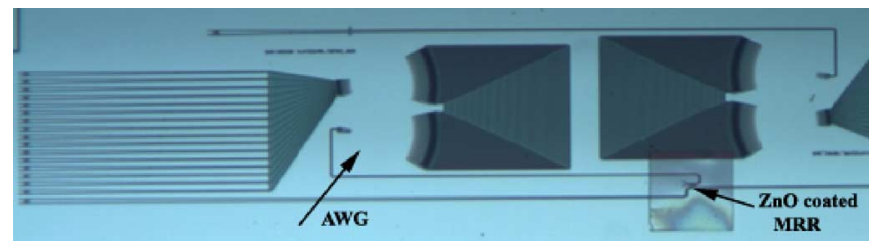

Fig. 2. Optical microscope image of AWG interrogated ethanol vapor sensor with $\mathrm{ZnO}$ porous film on the MRR. AWG used for spectral response characterization is partially shown in image.

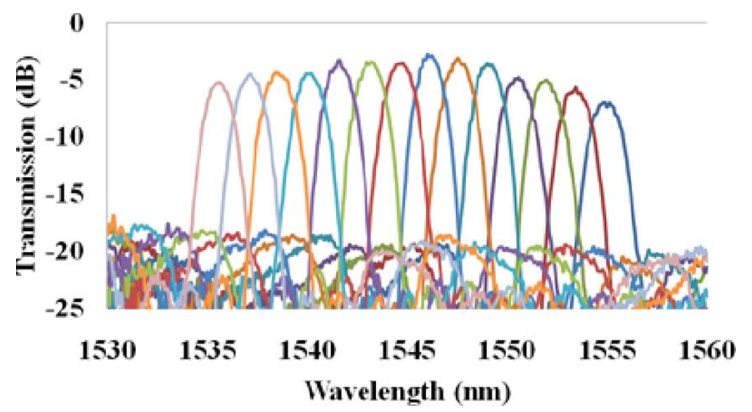

Fig. 3. Normalized transmission spectrum of AWG with $4 \mu \mathrm{m}$ wide input waveguide.

loidal nanoparticles suspended in ethanol. The porous nature of the film offers a large surface area for gas adsorption. The detailed nanoparticle synthesis and sensitive film preparation procedures are presented in [3]. Fig. 2 shows an optical microscope image of the fabricated AWG interrogated ethanol vapor sensor. For the purpose of preliminary studies on the AWG channel response, an additional AWG is fabricated on the same chip without a ring attached to it.

\section{EXPERIMENTS}

The spectral response from an AWG with a $4 \mu \mathrm{m}$ input waveguide measured with a superluminescent light emitting diode (SLED) and a spectrum analyzer is shown in Fig. 3. The transmission is normalized to that of a $450 \mathrm{~nm}$ wide photonic waveguide on the same chip. The insertion loss for the central channels is about $-3 \mathrm{~dB}$, and the nearest neighbor cross talk is around $-17 \mathrm{~dB}$, which is of a typical order for such compact SOI AWGs. Notably, a strong overlap between neighboring channels is achieved. The $3 \mathrm{~dB}$ bandwidth of a channel response is approximately $2.3 \mathrm{~nm}$ with over $0.9 \mathrm{~nm}$ overlap with channels at either side.

In the eventual sensing system, the broadband source and the detectors will be co-integrated with the sensing chip. However, for the present experiment the sensing chip is mounted on a temperature stabilized sample bed in a small gas chamber and the light source and the detector were kept outside. Vertical coupling through gratings is used to couple light in and out of the sensor. The top side of the gas chamber is covered with a glass window to facilitate the coupling. While a single mode fiber is used for in-coupling, the output light is collected by an Infrared Camera. Further details on the setup are presented in [3]. The measurement accuracy of this setup is highly influenced by the noise due to the light which is scattered from the sample surface and reaches the camera. Due to the typically low power spectral density of a broadband source, we have chosen to use a tunable laser source of about $3 \mathrm{~mW}$ to couple sufficient light through 

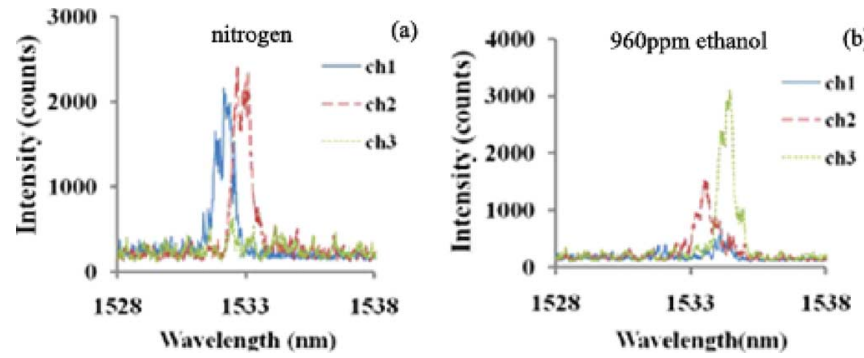

Fig. 4. Illustrations of intensity changes measured from three neighboring channels: (a) at 0 ppm (pure nitrogen) and (b) at $960 \mathrm{ppm}$ ethanol vapor concentrations.
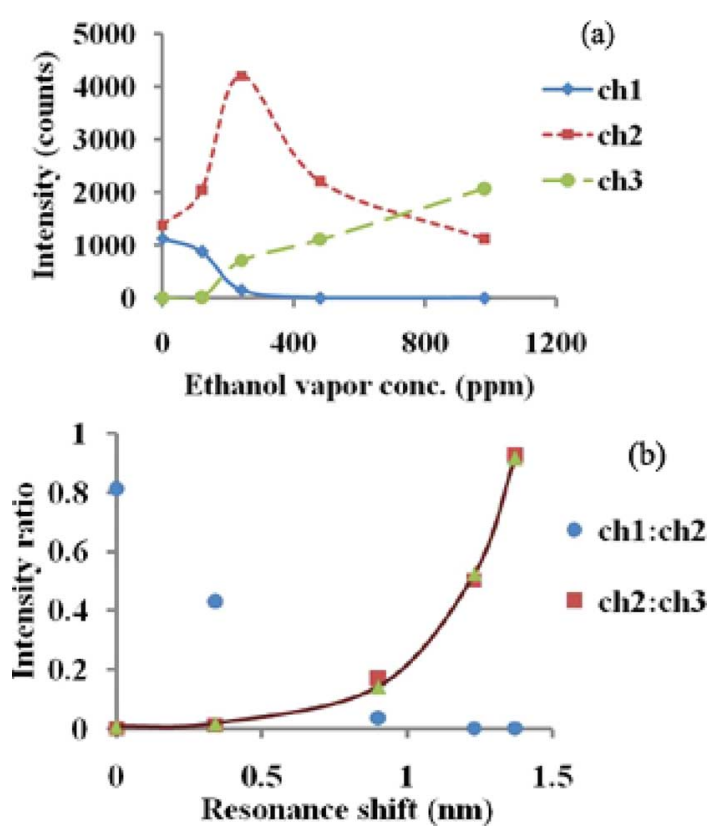

Fig. 5. (a) Average intensities measured from three channels at different ethanol vapor concentrations. (b) Calculated intensity ratios as a function of resonance shifts corresponding to vapor concentrations shown in (a) with exponential fit to $\operatorname{ch} 2: \operatorname{ch} 3$ (solid line).

the glass cover. Operating at such low powers reduces the noise on the camera measurement and allowed us to characterize the device in a gas environment. Fig. 4 illustrates how the intensity across adjacent channels changes with changing gas concentration at room temperature. The noisy features are due to the distant coupling through the glass window, and the scattered light.

The output intensities from three consecutive AWG channels are simultaneously monitored before and after exposure to 120 ppm, $240 \mathrm{ppm}, 480 \mathrm{ppm}$ and $960 \mathrm{ppm}$ ethanol vapor concentrations. The average intensity measured from each channel is then used to calculate the fractional intensity between adjacent channels at a given gas concentration. To better visualize the relation between the resonance wavelength and the intensity ratios, the resonance shifts at these vapor concentrations are recorded from the MRR through-port. Fig. 5(a) depicts the average channel intensities as a function of vapor concentration, and Fig. 5(b) shows the intensity ratios calculated at the corresponding measured resonance shifts. The solid line in Fig. 5(b) is an exponential fit to the ratios between channels 2 and 3 .
As observed in Fig. 5, fairly smooth transition from a pair of adjacent channels to the next pair is readily achieved signifying a good overlap between neighboring channels. More interestingly, the third channel emerges to take part in the play as the intensity ratio between the first two channels begins to fall below 0.2 , which is comparable with the simplified theoretical estimate for a $2 \mathrm{~nm}$ FWHM AWG as shown in Fig. 1. The FWHM of the resonance measured at the MRR through port is less than $50 \mathrm{pm}$. While our on-chip sensor-interrogator system is very suitable for highly sensitive gas sensors as demonstrated in this work, it can also be used to detect small resonance shifts. From the trend shown in Fig. 5(b), resonance shifts ranging from $50 \mathrm{pm}$ to $800 \mathrm{pm}$ should be readily interrogated with the ratio between just two channels.

In order to further ensure compact and low cost implementation of such a sensor, integrated approaches should replace external temperature stabilization mechanisms. A reference ring fabricated in series with the sensor can be used to monitor temperature driven wavelength drifts. Accordingly, the resonance wavelength of the reference ring can be designed to overlap with the AWG channels that are not used by the sensor. For athermal AWG operation, a cladding material with a negative thermo-optic coefficient of an appropriate magnitude can cover the sample except the rings.

\section{CONCLUSION}

We demonstrate that a compact on-chip AWG spectrometer can be used to interrogate resonance shifts from MRR sensors. An SOI MRR ethanol vapor sensor is interrogated by a 200 $\mathrm{GHz}$ AWG designed to have strongly overlapping output channels. Such an on-chip interrogation system presents itself as an attractive solution to the current interrogation challenges, and opens opportunities for low cost and compact implementation of MRR-based sensors.

\section{REFERENCES}

[1] M. Iqbal, "Label-free biosensor arrays based on silicon ring resonators and high-speed optical scanning instrumentation," J. Sel. Top. Quant. Electron, vol. 16, pp. 654-661, Jun. 2010.

[2] A. Ramachandran, "A universal biosensing platform based on optical micro-ring resonators," Biosensors Bioelectron., vol. 23, pp. 939-944, Sep. 2007.

[3] N. Yebo, "An integrated optic ethanol vapor sensor based on a silicon-on-insulator microring resonator coated with a porous $\mathrm{ZnO}$ film," Opt. Expr., vol. 18, pp. 11859-11866, May 2010.

[4] W. Bogaerts, "Silicon-on-insulator spectral filters fabricated with CMOS technology," J. Sel. Top. Quant. Electron., vol. 16, pp. 33-44, Feb. 2010.

[5] H. Su, "A novel fiber Bragg grating interrogating sensor system based on AWG demultiplexing," Opt. Commun., vol. 275, pp. 196-200, Feb. 2007.

[6] P. Cheben, "Tilted fiber Bragg grating sensor interrogation system using a high-resolution silicon-on-insulator arrayed waveguide grating," Opt. Lett., vol. 33, pp. 2647-2649, Nov. 2008.

[7] H. Guo, "Interrogation of a long-period grating sensor by a thermally tunable arrayed waveguide grating," IEEE Photon.Technol. Lett., vol. 20, pp. 1790-1792, Nov. 2008.

[8] Q. Fang, "Compact SOI arrayed waveguide grating demultiplexer with broad spectral response," Opt. Commun., vol. 258, pp. 155-158, Jul. 2005. 